Tight Junctions: From simple barriers to multifunctional

molecular gates

Ceniz Zihni ${ }^{1}$, Clare Mills ${ }^{1}$, Karl Matter $^{1 \#}$ and Maria S Balda ${ }^{1 \#}$

\author{
Department of Cell Biology \\ UCL Institute of Ophthalmology \\ University College London \\ London EC1V 9EL, UK
}

\# Address for correspondence

UCL Institute of Ophthalmology

University College London

Bath Street

London EC1V 9EL

United Kingdom

Tel - 44207608 4014/6861

Fax - 442076084034

E-mail:k.matter@ucl.ac.uk/m.balda@ucl.ac.uk

Main body of text: 5983 words 
Epithelia and endothelia separate different tissue compartments and protect multicellular organisms form the outside world. This requires the formation of tight junctions, selective gates that control paracellular diffusion of ions and solutes. Tight junctions also form the border between the apical and basolateral plasma membrane domains and are linked to the machinery that controls apicobasal polarization. Additionally, signalling networks that guide diverse cell behaviours and functions are connected to tight junctions, transmitting information to and from the cytoskeleton, nucleus and different cell adhesion complexes. Here, we discuss recent advances in our understanding of the molecular architecture and cellular functions of tight junctions.

Microscopists in the $19^{\text {th }}$ century described the paracellular space between neighbouring cells within an epithelial sheet to be sealed by a "terminal bar", a structure later resolved by electron microscopy into a composite of distinct cell-cell junctions that is now called the epithelial junctional complex and is formed by tight junctions, adherens junctions and desmosomes ${ }^{1,2}$. As the former two junctions are more tightly associated and often reside at the apical end of the lateral membrane, they are often referred to as the apical junctional complex (however, in endothelia, tight junctions and adherens junctions can be intercalated) (Fig. 1). Tight junctions are essential for barrier formation, and their primary physiological role is to function as paracellular gates that restrict diffusion on the basis of size and charge. Selective paracellular diffusion is an essential process for the maintenance of homoeostasis in organs and tissues. Tight junctions have long been the most enigmatic of all adhesion complexes and eluded a detailed molecular and functional analysis due to their complex architecture. Recent years have witnessed the identification of a large array of components associated with tight junctions implicating these junctions in an unexpected range of different functions, thereby challenging the traditional model, in which tight junctions are considered a simple diffusion barrier formed by a rigid molecular complex. In line with these various functions, mutations in genes encoding tight junction proteins have been linked to a range of inherited human diseases. Additionally, tight junction components are known to be targeted by a number of pathogenic bacteria and viruses, which hijack tight junction proteins to enter and infect cells, or target junctional signalling mechanisms to cross tissue barriers. Although tight junctions are a vertebrate junction, many of their components and functions are evolutionarily conserved (Box 1).

The main purpose of this review is to examine the recent advances in the unravelling of the molecular architecture of tight junctions and understanding their functions. We will discuss recent exciting insights into how tight junctions function as signalling platforms that guide cell behaviour and differentiation, as well as their role in cell polarization. We will also survey recent results suggesting unexpected crosstalk between tight junctions and other adhesive structures.

\section{Structure and composition}

Electron microscopy revealed that tight junctions form very close focal contacts between plasma membranes of neighbouring cells that, depending on the preservation method used, may appear as apparent hemifusions or "kisses" ${ }^{2}$ (Fig. 1). By freeze fracture electron microscopy, a technique that enables the imaging of the hydrophobic interior of a membrane, tight junctions appear as meshwork of fibrils, often apparently formed by rows of transmembrane particles, that are thought to represent the diffusion barriers ${ }^{3,4}$. Tight junctions also contain an electron dense junctional plaque that consists of cytosolic proteins that form the interface between the junctional membrane and the cytoskeleton. 


\section{Transmembrane proteins}

The main protein components of the transmembrane strands observed by freeze fracture electron microscopy are tetraspan proteins of the claudin family (encompassing 26 members in humans and 27 in mouse, see below) and the three junctional MARVEL domain proteins: occludin, tricellulin and MarvelD3. Although not confirmed for all members of the two families, these proteins have been localised by immunoelectron microscopy to the strands and shown to induce superficially similar strands if expressed in cells that do not form tight junctions in the case of claudins or transmembrane particles and short strand fragments in the case of occludin ${ }^{5-8}$. Similarly, some claudins and occludin are able to mediate Ca-independent cell-cell adhesion, further supporting a model in which tight junctions consist of multimeric transmembrane protein complexes that mediate cell-cell adhesion ${ }^{8,9}$.

Other transmembrane components of tight junctions include a trispan protein, BVES (blood vessel epicardial substance), and a large group of single span transmembrane adhesion proteins with two immunoglobulin like domains that comprises junctional adhesion proteins (JAMs), Coxsakie adenovirus receptor (CAR), and angulins (or lipolysis-stimulated lipoprotein receptors) ${ }^{10-}$ 15. Angulins, together with tricellulin, are enriched in tricellular corners and are thought to mediate functional integrity of the junction in epithelial and endothelial cells. Additionally, Crb3, a protein with EGF like domains important for apical polarization, associates with tight junctions ${ }^{16,17}$. These proteins have not been shown to associate with the transmembrane strands but can modulate the strength of the junctional barrier if removed or overexpressed, or, like JAM proteins, regulate junction assembly (see below). Many of the functions of transmembrane tight junction proteins depend on interactions with components of the complex cytosolic plaque that lines the junctional membrane.

\section{Junctional plaque components}

The cytosolic plaque is a complex protein network that interacts with the cytoplasmic domains of junctional membrane proteins as well as with the F-actin and tubulin cytoskeleton (Fig. 1B). Its main structural components are adaptor proteins that contain multiple protein/protein interaction motifs $^{18}$. A typical example is the first tight junction protein identified, ZO-1 (zonula occludens-1), a $220 \mathrm{kDa}$ peripheral membrane protein, consisting of an N-terminal half with three PDZ (PSD-95, DIgA, ZO-1 homology) domains, an SH3 (Src homology 3) domain, and a GUK (yeast guanylate kinase homology domain); whereas its $\mathrm{C}$-terminal half interacts with $\mathrm{F}$-actin and contains alternatively spliced domains that may confer tissue-specific functions ${ }^{19-21}$. The $\mathrm{N}$-terminal motifs bind to different transmembrane proteins: claudins via the first PDZ domain, JAMs via the third and occludin via the GUK domain. The SH3 domain links ZO-1 to junctional signalling mechanisms by binding to ZONAB (see below), a transcriptional and posttranscriptional regulator of gene expression, and Apg-2, a heat shock protein ${ }^{20,22-25}$. ZO-1 also engages in intramolecular interactions that lead to a closed conformation in which access to the central GUK/SH3 module is limited $^{26,27}$. A feature that may also underlie the inability of in vitro translated full length ZO-1 to bind $\mathrm{SH} 3$ domain ligands that bind to shorter domain constructs ${ }^{28}$. Although the functional relevance of such intramolecular interactions is poorly understood, they may serve as regulatory switches for ligand binding and, thereby, junction formation and signalling.

The tight junction plaque contains a vast number of such adaptor proteins, many interacting with each other, forming a protein network. Examples include ZO-2 and ZO-3 two proteins that coimmunoproecipitate with ZO- $1^{29-31}$. They have the same domain structure as ZO- 1 within the $\mathrm{N}$ terminal half but have unique C-terminal parts. ZO-1 and ZO-2 interact with ZO-1 in a mutually exclusive manner via the second PDZ domain. Other examples are the MAGI proteins, which have the same type of domains as the ZO proteins but in an inverted arrangement, or the multi-PDZ domain proteins MUPP1 and PATJ ${ }^{32-36}$. Another type of junctional adaptor is represented by 
cingulin and JACOP/paracingulin, two homologous coiled-coil proteins that bind to a variety of different junctional proteins, including $\mathrm{F}$-actin and, in the case of cingulin, microtubules ${ }^{37,38}$. The cytoplasmic plaque may be an ordered structure with proteins like cingulin being farther from the membrane than core proteins like ZO-1 (Fig. 1C) ${ }^{39}$. Although such proteins may have a distinct overall distribution within the junction, their distributions overlap and they are able to interact with each other and form complexes ${ }^{40}$. The junction also contains many different signalling proteins that are recruited by binding to adaptors or to membrane proteins such as occludin and MarvelD $3^{41,42}$. This includes protein kinases, phosphatases, monomeric and trimeric GTP-binding proteins and transcriptional and post-transcriptional regulators, which participate in various signalling pathways $(\text { Table } 1)^{43-45}$.

The number of junctional proteins, many of which belonging to the same protein family or having similar protein binding motifs, is intriguing. While the many claudins can be explained by their different functional properties, it is more difficult to understand why so many adaptor proteins are required. Knockouts and knockdowns in cells in culture or whole organisms yielded often only minimal functional consequences (e.g., cingulin, ZO-3) ${ }^{46,47}$. Hence, many junctional proteins are considered to have redundant functions. However, this is surely not the case for ZO-1 and ZO-2, as individual knockouts of both adaptors are embryonic lethal ${ }^{48,49}$. Even in vitro, the lack of a clear phenotype in loss of function experiments studying ZO-1 in MDCK cells was later attributed to incomplete depletion in some RNA interference studies as reexpression of ZO-1 at low levels in knockout cells could rescue the phenotype ${ }^{50}$. Nevertheless, adaptive processes in the junctional protein network in response to removal of individual components do occur. For example, deletion of ZO-3 in mice leads to increased junctional recruitment of ZO- $2^{46}$. Such adaptive processes render junctions biologically more robust, but they make experimental analysis more difficult as, for example, constitutive depletion methods in cell lines may lead to adaptive processes during cell line selection. The apparent redundancy under standard experimental conditions may also mean that a protein is only important under certain conditions or in specific cell types and/or model systems, as exemplified by the importance of ZO-3 for osmoregulation in zebrafish or occludin for barrier maintenance in ethanol-stressed mice ${ }^{51,52}$. Hence, understanding the functional role of specific tight junction proteins and how they cooperate to form functional junctional complexes will require a more detailed analysis of specific tissues and processes in different experimental models, conditional gene inactivation approaches, overexpression experiments, and analysis of how junctional complexes adapt to removal of specific components.

\section{Models of tight junction structure}

The ultrastructural appearance of tight junctions has stimulated the discussion about their molecular architecture for decades. Two models have emerged: a protein model and a protein/lipid hybrid model; the latter is often referred to as the lipid model, although the need for proteins had always been accepted (Fig. 2A). The protein model posits that the paracellular diffusion barrier is formed by transmembrane proteins that form an intercellular protein complex between the two neighbouring plasma membranes that have a standard bilayer lipid configuration. This model has recently gained more support due to the identification of the claudin crystal structure and subsequent modelling approaches that enabled the construction of a model that provides a good fit to the junctional ultrastructure. The $2.4 \AA$ resolution structure of claudin- 15 revealed a characteristic $\beta$-sheet fold formed by the two extracellular domains, which are anchored to a transmembrane four-helix bundle (Fig. 3) ${ }^{53}$. Claudin-15 forms a linear polymer mediated by specific interactions between adjacent extracellular domains that are required for reconstituting tight junction-like intramembrane strands. Cysteine crosslinking then led to a model in which two such anti-parallel strands associate with each other to form an intramembrane tight junction-strand ${ }^{54}$. Consequently, 
each membrane-membrane contact site consists of four claudin polymers, two per cell, that interact via their extracellular domains, forming the junctional barrier and permeation pathway. As different claudins may either polymerize within the same strand or adjacent strands in a heterotypic or homotypic fashion, tight junction strands form a mosaic consisting of various claudin molecules ${ }^{55-57}$. While this model provides a possible structural explanation for how claudins may form linear strands, it is more difficult to see how other junctional membrane proteins that associate with strands (e.g., occludin) and have different dynamic properties fit into this model ${ }^{58}$. Similarly, native junctional intramembrane strands are not linear but anastomose, leading to the formation of networks (Fig. 1E). Hence, a molecular model for strand formation requires a mechanism for branching.

The hybrid model offers possible answers to some of these questions. It proposes that the close membrane-membrane contact sites are actual membrane hemifusions and that the intramembrane strands are cylinders of inverted lipid micelles. In this case, the lamellar structure of the membrane lipid bi-layer forms a hexagonal transitory arrangement whereby lipid chains are oriented outwards (Fig. 2A $)^{59,60}$. This model is based on the demonstration that protein-free liposomes can form tight junction-like strands ${ }^{61}$. A hemifusion state is energetically unfavourable; hence, transmembrane proteins were proposed to stabilise the inverted micellar structure ${ }^{59}$. This model is supported by the observation that native tight junction strands seem to contain protein and phospholipids ${ }^{62,63}$. According to this model, lipids are filling the gaps between different types of membrane proteins, eliminating the need of proteins forming continuous polymers ${ }^{58,64}$. Consequently, this model enables to explain why different protein components that have different dynamic properties can be a part of the same strands.

Different experiments have been performed to differentiate between the two models, but a consensus was not reached. Cell-to-cell lipid diffusion experiments should provide an answer as lipids can only diffuse from one cell to another if the exoplasmic leaflet of two neighbouring cells is continuous as in the hybrid model. However, lipids with large polar heads did not diffuse whereas fluorescently labelled lipids with modified acyl chains $\mathrm{did}^{65,66}$. It is thus possible that alternative barriers prevent diffusion of lipids with large head domains (e.g., the negative membrane curvature) or that the modified lipid exchanges between closely apposed membranes. Regardless of the model, lipids are important for tight junctions. For example, some tight junction proteins are associated with cholesterol-rich, detergent-resistant membrane microdomains and modification of cholesterol modifies epithelial barrier properties ${ }^{67-69}$. However, freeze-fracture analysis of tight junctions of cholesterol depleted cells yielded contradictory results, and cholesterol may affect a membrane by influencing the lipid structure or the functional properties of transmembrane proteins by altering their lipid environment ${ }^{70,71}$. Finally, one could also imagine that the strands are not as homogenous as generally assumed and might be composed of sections containing different lipid structures and protein composition.

The true structure of tight junctions thus remains to be established. As intramembrane strands can thus far only be seen in fixed specimen, new methods will have to be developed that enable visualization of strand dynamics. Hopefully, improvements of live imaging approaches combined with enhanced resolution of light microscopy may provide the key to answer these questions. Nevertheless, the current models and structural data allow us to gain a better understanding of how tight junctions function as permeability barriers.

\section{Barrier functions}

Tight junctions form two types of barriers: a paracellular one that regulates selective paracellular permeability and an intramembrane one that restricts exchange of membrane components between the apical and basolateral cell surface domains (Fig. 2B). The two barriers have the same physical location at intramembrane strands and are likely to be structurally related. 


\section{Regulation of paracellular permeability}

The physiological properties of the junctional gate are that of a semipermeable diffusion barrier that discriminates solutes on the basis of size and charge ${ }^{72}$. Solutes can cross the junctional paracellular pathway along two routes. A charge-selective permeation pathway that is thought to consist of pores across serially arranged barriers allowing diffusion of ions and small uncharged molecules (Fig. 2C). These pores have a diameter of $\sim 4-8 \AA$ depending tissue and molecule analysed $^{73-75}$. A second diffusion pathway allows the diffusion of larger solutes, macromolecules up to a size limit of $\sim 30-60 \AA^{72,75}$. Charge-selective ion permeation and size-selective macromolecular diffusion occur by different mechanisms and can be regulated in opposing manners ${ }^{76}$. Ion permeation is typically measured by assessing electrical resistance or conductivity, an instantaneous measurement that requires a continuous conductive pathway for a current to flow. Macromolecular diffusion is slow, requires tracer diffusion measurements over longer periods of time (i.e., hours), and, hence, may occur in a stepwise manner (Fig. 2D). To explain chargeselective permeation, a model of the junction evolved that considers the intramembrane strands to contain regulated ion-selective channels that can open and close. To account for macromolecular permeation a dynamic strand model has been introduced, in which the intramembrane strands remodel, allowing slow, diffusion (Fig. 2D). Such serial diffusion barriers might be formed by protein polymers according to the protein model or, alternatively, inverted lipid cylinders whose stability is regulated by associated proteins ${ }^{76}$. In this model, the size-selectivity would be determined by the distance between the outer leaflets of the two adhering plasma membranes. In an alternative model, tight junctions were proposed to be formed by two differently sized inverted micelles and macromolecular diffusion to occur inside the larger ones that dynamically form and dissolve ${ }^{75}$. However, current freeze fracture data does not suggest that junctions contain two different sizes of intramembrane strands.

While significant progress has been made in the elucidation of the molecular mechanisms that enable junctional ion permeability, macromolecular diffusion is still not well understood and currently available insights have been summarized in other reviews ${ }^{75,76}$. Hence, we will focus here on recent exciting findings deciphering the molecular mechanisms underlying junctional ion permeation.

Upon their discovery, claudins quickly emerged as candidates for mediating ion-selective paracellular diffusion, as one of them, claudin-16 (paracellin-1) was identified as a gene mutated in renal magnesium wasting, an inherited disease affecting renal paracellular magnesium reabsorption (Box 2$)^{77}$. Claudins' tissue-specific expression pattern further fuelled the hypothesis that the claudin composition of a tight junction determines the permeability properties. Claudins are now grouped according to their channel- and barrier-forming properties into those that support cation-selectivity (e.g. claudin-2, 10b, 15), anion-selectivity (e.g. claudin-10a, 17), or preferentially sealing claudins (e.g., claudin-1 $)^{73,78-85}$. The latter group are claudins that have not (yet) been associated with promoting permeability of a specific type of ion or molecule, and are hence thought to enhance the barrier function. However, it is also possible that they form pores for yet to be identified molecules.

Expression studies combined with measuring permeability of epithelial cell monolayers demonstrated that claudins are important determinants of the paracellular barrier properties. First evidence for channel formation came from structural and functional studies demonstrating that modification of the first extracellular loop of claudins affects the conductive properties of claudintransfected cells ${ }^{85,86}$. For example, the cation-selective claudin- 2 contains a critical residue in its first extracellular loop that, if replaced with a cysteine followed by modification with the thiolreactive bulky reagent methanethiosulfonate was found to block the ion-conductive pathway ${ }^{87,88}$. 
More direct evidence came from a recent patch clamp approach demonstrating that claudin-2 indeed forms a gated cation-selective paracellular pore ${ }^{89}$.

The recent x-ray crystal structure of claudin-15, a cation-selective claudin, provides a possible structural basis to explain the formation of ion-selective channels ${ }^{53,54,90,91}$. According to the model proposed, a characteristic anti-parallel $\beta$-sheet fold formed by the first extracellular loop and the Cterminal end of the second extracellular loop is anchored to a conserved transmembrane four-helix bundle (Fig. 3A). Apart from segment 3 , the length of the transmembrane domains is consistent with the lipid bilayer thickness and they contain residues with small side chains that are important for tight helical packing. Mutations leading to changes in such residues are associated with human disease, suggesting that they are indeed important ${ }^{77,92}$. Similarly, mutation of the two conserved cysteine residues in extracellular loop 1 abolishes the barrier forming ability of claudin-5, suggesting that the disulfite bond is important to stabilise the $\beta$-sheet structure ${ }^{93}$. Head-to-head association of two antiparallel claudin strands from one cell with two strands from the neighbouring cell is thought to lead to the formation of a $\beta$-barrel that defines the paracellular pore (Fig. $3 B, C)^{54}$. Other authors have expressed reservations about the packing density generated by such a structure ${ }^{94}$. However, the $\beta$-barrel model is compatible with structure-function studies performed previously. For example, the negatively charged residues Glu55 and Asp64 of claudin-15 extend away from the $\beta$-sheet surface, leading to a negatively charged $\beta$-barrel, which would be compatible with a cation-selective pore. Previous work indeed demonstrated that substituting these two residues by positively charged ones alters ion-selectivity of this claudin ${ }^{86}$. Similarly, homology models indicate that cation-selective and anion-selective claudins- 2 and -10 a form negatively and positively charged barrel surfaces, respectively ${ }^{53}$. Altogether, this $\beta$-barrel model provides an excellent base for future work to elucidate the structural basis for junctional ion permeation.

Patch clamp experiments indicate that the junctional pores are gated, but the structural basis for opening and closing has not been analysed ${ }^{89}$. Gating might be regulated by mechanisms that are yet to be identified. An interesting paradigm is provided by WNK1 and WNK4, two kinases linked to Pseudohypoaldosteronism type II, an autosomal dominant disorder that leads to hypertension. WNK4 localises to tight junctions and expression of disease-causing alleles of the two kinases stimulates phosphorylation of multiple claudins and leads to increased chloride permeability ${ }^{95-99}$. While these studies indicate that claudin-mediated ion conductance is regulated, the structural changes that lead form phosphorylation of claudins to opening claudin-based pores remain to be determined.

\section{Intramembrane diffusion barrier: the fence function}

The junctional fence has been defined by diffusion experiments with fluorescent lipid probes and lipids that demonstrated that a diffusion barrier restricts intermixing of apical and basolateral lipids in the exoplasmic plasma membrane leaflets (Fig. 2B) ${ }^{100,101}$. Although not directly demonstrated, based on these properties one would assume tight junctions also act as a fence for transmembrane proteins. The fence function is assumed to be linked to the intramembrane strands as experimental manipulations that do or do not affect their integrity often have analogous effects on integrity of the junctional fence. For example, transient ATP depletion in MDCK cells has no effect on the fence function and the suprastructure of tight junctions but, for unknown reasons, leads to disruption of the paracellular diffusion barrier ${ }^{102}$. In contrast, treatment of epithelial cells with a rotavirus derived peptide leads to a partial disruption of the continuous intramembrane strand network and increased lipid diffusion between apical and basolateral plasma membrane domains $^{103}$. In the protein model, the transmembrane proteins forming the strands serve to restrict intramembrane diffusion; whereas in the hybrid model, apical and basolateral membrane leaflets are discontinuous, inherently generating an exoplasmic fence. However, also here one would expect transmembrane proteins to play a role, as the unconventional lipid structures of the hybrid 
model require stabilisation (Fig. 2A). Expression of an occludin mutant with inactivated cytosolic domains that is unable to interact with the cytoplasmic plaque indeed disrupts the lipid diffusion barrier ${ }^{104}$. It does so without disrupting the network of intramembrane strands, arguing against a model in which the strands act as the diffusion fence. As this mutant form of occludin also results in increased macromolecular paracellular diffusion, it is possible that the fence function and the mechanism enabling macromolecular diffusion are related. According to the dynamic strand model, intramembrane strands remodel to allow macromolecular diffusion (Fig. 2D). If the strands were responsible for the fence function as well, increased strand dynamics would indeed lead to increased intramembrane and macromolecular diffusion. Testing such a model will require the development of approaches to visualise both lipid diffusion and intramembrane strand dynamics in live cells.

The overall importance of the fence for maintaining epithelial polarity is often overestimated as cells that have a defective junctional fence due to expression of mutant form of occludin still polarize $^{104}$. Even cells that lack tight junctions due to combined removal of ZO-1 and ZO-2 still polarize and maintain at least some polarized lipid distribution ${ }^{105,106}$. It remains thus to be established to what extent the fence function is physiologically required for functional epithelia.

\section{Assembly and links to apical polarity}

Establishment of tight junctions is a multistep process that involves an array of distinct signalling mechanisms that guide and control this process, and ultimately leads to cell surface polarization and the differentiation of often organ-specific apical domains, such as the intestinal brush border membrane.

\section{Tight junction assembly}

Tight junction assembly is commonly studied in Ca-switch models in which cells are plated on a filter, enabling transepithelial measurements for barrier formation, in a medium with a low calcium concentration that does not allow junction formation, which is then induced by adding additional calcium $^{107,108}$. Such experiments demonstrated that tight and adherens junctions are not morphologically well-defined during early junction assembly but resemble the primordial junctional complexes observed in primitive nematodes and lie within the same plane as the basal, focal adhesion complex (Fig. 4A). The close relationship between tight and adherens junctions is reflected in biochemical interactions between core components of these junctions. For instance, ZO-1 and $\alpha$-catenin, a protein that links adherens junctions to the actin cytoskeleton, form a complex in cells grown in low calcium; upon initiation of junction formation, this ZO-1- $\alpha$-catenin complex is recruited to forming junctions, coupling assembly of tight and adherens junctions (Fig. $4 A)^{109,110}$. A central coordinating role is also played by nectins, adherens junction adhesion proteins that participate in the recruitment of JAM-A ${ }^{111}$. JAM-A localizes first to nectin-based cell-cell contacts, which is dependent afadin forming a bridge between nectin and ZO-1, a JAM-A-binding protein. Formation of mature tight and adherens junctions then requires activation of multiple signalling mechanisms that include different PKCs, PKA, AMPK, protein phosphatases, and small and heterotrimeric GTPases (Table 1) ) $^{43-45,108,112}$.

Many tight junction proteins interact with the actin cytoskeleton. Although the importance of individual interactions is still poorly understood, regulation of cytoskeletal dynamics is essential for junction formation and function. For example, myosin light chain kinase, a regulator of actomyosin activity, stimulates increased intestinal paracellular permeability during inflammation, a process involving junctional remodelling and occludin internalization ${ }^{113}$. RhoGTPases are major regulators of the actin cytoskeleton and, consequently, play fundamental roles in the regulation of junction assembly and function. The mechanisms that control RhoGTPase signalling have been intensively investigated during the last years, leading to the discovery of regulators that control specific 
processes by guiding the activity of RhoA, $\mathrm{Cdc} 42$, and $\operatorname{Rac}^{43,45}$. This includes guanine nucleotide exchange factors (GEFs) for RhoA, p114RhoGEF/ ARHGEF18 and ARHGEF11, that are recruited to forming junctions by cingulin, JACOP, and ZO-1, respectively, to promote ROCK driven myosin activation and junction formation (Fig. 4A) ${ }^{114-116}$. Similarly, the Cdc42 GEF TUBA is recruited to tight junctions by ZO-1 and tricellulin and regulates the junctional actomyosin cytoskeleton ${ }^{117,118}$. How these different mechanisms are coordinated with each other and are integrated into the cellular signalling networks that guide cell behaviour is still poorly understood and remains to be investigated in more detail.

\section{Establishment of apical polarity}

Establishment of tight junctions is intimately linked with the signalling mechanisms that drive epithelial polarization ${ }^{112,119}$. During the initial assembly of junctions, the adhesion protein JAM-A recruits the PAR3-PAR6-aPKC complex ${ }^{120,121}$; thereby establishing the forming border between the apical and lateral domains (Fig. 4A, Table 1). The PAR3-PAR6-aPKC complex is an evolutionarily conserved signalling module that drives apical polarization in response to Cdc42 activation. It is thought that early steps of junction formation are supported by the Cdc42 GEF ECT2 that associates with PAR3 and PAR6, and requires interplay with the GTPase activating proteins Rich1 and SH3BP1 that complete the GTPase cycle ${ }^{122-124}$. Once cells start to polarize, activation of Cdc42 at the apical pole and the apical margin close to tight junctions is catalysed by the GEF Dbl3, which is recruited by another pro-apical signalling determinant, ezrin ${ }^{125}$. Apical Cdc42 activation then promotes aPKC activation, leading to phosphorylation and dissociation of PAR3 from the PAR6aPKC complex. The latter translocates to the differentiating apical membrane in a still poorly understood process, whereas PAR3 remains at tight junctions and marks the border between apical and lateral domains. This process is essential for the development of specialised apical membrane domains, such as the intestinal brush border membrane, and drives the accumulation of apical signalling proteins (e.g., Crb3) as well as proteins required for apical functions (e.g., brush border enzymes $)^{125}$. This mechanism is evolutionarily conserved and linked to the subapical zone and adherens junctions in Drosophila (Box 1) ${ }^{126,127}$.

A second pro-apical signalling complex also associates with tight junctions, the Crb3/Pals1/PATJ complex, which links to tight junctions via PATJ interacting with ZO-3, claudin-1 and JAM$A^{128-130}$. Crb3 is a transmembrane protein that during apical differentiation is phosphorylated by aPKC, which involves an interaction with the PAR6 ${ }^{131}$. Activation of pro-apical signalling does not only promote apical differentiation, it also leads to a suppression of pro-basolateral determinants $^{119,132}$. Consequently, the extent of apical Cdc42 activation also determines the relative size of the two cell surface domains and, thereby, the positioning of the tight junction, the apical-lateral border ${ }^{125}$.

\section{Signalling from tight junctions}

It has become apparent that tight junctions, apart from serving as permeability barriers, are also important signalling platforms. As discussed above, assembly of these junctions is inherently linked to the establishment of epithelial apicobasal polarity. Additionally, tight junctions transmit signals to the cell interior to regulate the cytoskeleton, gene expression, cell proliferation and differentiation during various cellular processes (Table1). These mechanisms have recently been reviewed and we will only summarize some of the central principles and recent developments ${ }^{43,44,112}$.

To start, tight junctions signal to guide cell proliferation and differentiation. Their formation accompanies the establishment of epithelial sheets with the increasing cell density inhibiting proliferation. This process includes well-known signalling mechanisms such as the Hippo pathway that regulates the transcriptional coactivators YAP and TAZ. Tight junction associated mechanisms that affect activity of these transcription factors includes both junctional recruitment of Hippo 
pathway kinases that phosphorylate and inhibit them, the merlin tumour suppressor mechanism that links the Crb signalling complex to inhibition of proliferation, as well as interactions with junctional adaptor proteins such as angiomotin, ZO-2 and PAR3 ${ }^{133-138}$. Other proliferation-regulating transcription factors have been shown to localise to tight junctions as well as nuclei, such as ZONAB, a protein inhibited by ZO-1 binding that promotes proliferation and interacts with other proteins at cell junctions such as symplekin, RalA, GEF-H1 and CDK4 ${ }^{23,24,139-142}$. As ZONAB binds multiple proteins at tight junctions, removal of ZO-1 alone may not be sufficient to lose junctional localisation as has recently been suggested ${ }^{28}$. The latter paper also concluded that ZO-1 does not bind $Z O N A B^{28}$. However, others who employed the same extraction conditions as originally described for the isolation of ZO-1/ZONAB complexes co-immunoprecipitated the two proteins successfully, and suggested a molecular and functional link with claudin-2 $2^{143-145}$. The ZO-1/ZONAB pathway is thought to regulate expression of ErbB2, cyclin D1 and PCNA ${ }^{23,146}$. This has recently been questioned as no effect on the expression of these genes was detected in MDCK cells constitutively depleted of ZO-1, ZO-2 and ZO-3 individually or together ${ }^{28}$. However, these assays were performed in low density, proliferating cells in which ZONAB is fully active and not inhibited by ZO-1 (even overexpression of ZONAB does not stimulate proliferation in such cells) ${ }^{139}$. Recent studies linked the ZO-1/ZONAB pathway to distinct regulatory mechanisms. CFTR, the transmembrane protein linked to cystic fibrosis, binds and stabilises ZO-1, leading to reduced ZO-1 expression in its absence and, thereby, promoting nuclear translocation of ZONAB, induction of cyclin D1 and repression of ErbB2 ${ }^{147}$. Manipulation of other junctional transmembrane proteins, such as BVES, regulates ZONAB activation via a GEF-H1/RhoA-stimulated mechanism ${ }^{140,148}$. In endothelial cells of the blood-tumour-barrier, bradykinin-induced activation of nitric oxide synthesis induces increased permeability and ZONAB activation, leading to repression of claudin-5 and occludin promoters $^{149}$. The group of dual localization proteins associated with tight junctions also includes junctional adaptors such as ZO-2, which travels to the nucleus at low cell density and interacts with several transcription factors including c-Myc, AP-1 and YAP, regulators of cell proliferation $^{44}$. Depletion of ZO-2 in MDCK cells induces cell size increases and enhanced cyclin D1 expression due to modulation of the YAP and Akt/mTOR pathways ${ }^{150}$.

Tight junctions can also signal to the cell interior via classical signalling cascades, such as the JNK1/2 mitogen activated protein kinase pathway that is regulated via an interaction between the membrane protein MarvelD3 and MEKK1 (MAP3K1) ${ }^{41}$. This pathway regulates epithelial cell proliferation and migration with MarvelD3 functioning as a dynamic attenuator that recycles between tight junctions and endosomes. Occludin, a close relative of MarvelD3, also interacts with a mitogen activated protein (MAP) kinase pathway, but suppresses dissociation of cell junctions by Raf-1 activated Erk signalling via unknown mechanisms ${ }^{151}$. Occludin interacts with multiple signalling proteins that may play a role and has also been linked to TGF $\beta$-induced junction dissociation (Table 1) (2,152,153. $^{\text {. }}$

Signalling at tight junctions seems to play an important role in the cellular stress response, and $\mathrm{ZO}$ proteins have been linked to junctional integrity in response to stress in zebrafish and $\mathrm{C}$. elegans, possibly due to regulating $\mathrm{F}$-actin remodelling ${ }^{51,154}$. Junctional processes also affect general cell behaviour during stress, such as the above-described MarvelD3-MEKK1-JNK1/2 pathway, which is important for cell survival during hyperosmotic stress. Similarly, stress-induced Erk activation stimulates the GEF-H1/ZONAB pathway to regulate gene expression posttranscriptionally as part of a Ras effector pathway that regulates cell survival ${ }^{142,155}$. In response to heat shock, ZONAB is activated by Apg-2, a heat shock protein that competes for ZONAB binding to ZO- ${ }^{24}$. Hence, tight junctions appear to function as sensors for cell stress and are components of MAP kinase signalling networks that can regulate MAP kinase activation as well as transmit MAP kinase signals. 


\section{Crosstalk with other adhesion complexes}

While different adhesion complexes that mediate interactions with neighbouring cells and the cell matrix are often considered in isolation, they influence each other strongly. As we discussed above for junction assembly, such crosstalk can involve formation of complexes containing components of different adhesion complexes. However, it can also involve regulation of signalling pathways (Fig. 4B). For example, JAM-A-mediated cell-cell adhesion signals via two related small GTPases of the Ras-related protein family: Rap1 and Rap2. JAM-A stimulated Rap2 activation promotes stabilisation of adherens junctions; and activation of Rap1 affects adhesion to extracellular matrix and cell migration by regulating integrin $\beta 1$ expression and recycling ${ }^{156-159}$. In endothelial cells, ZO-1 regulates overall cell-cell tension as well as tensile forces acting on adherens junctions by regulating recruitment of a complex formed by $p 114$ RhoGEF and JACOP, which stimulates junctional RhoA/ROCKII/myosin activation ${ }^{116}$. Loss of ZO-1 also promotes formation of stress fibres and focal adhesions indicating that signalling at tight junctions has cellwide consequences on the cytoskeleton and adhesion. Orchestration of cell-cell tension and focal adhesion formation by ZO-1 is functionally important for the regulation of cell migration and angiogenesis. Stress fibre formation and focal adhesion formation also depends on GEF-H1, which stimulates RhoA along the basal membrane and drives focal adhesion formation in various cell types. GEF-H1 is recruited to tight junctions by cingulin, which then leads to inhibition of the GEF, similar to the inhibition of this GEF by binding to microtubules (Table 1$)^{160-165}$. While a role for ZO-1 in suppressing focal adhesion formation has so far only been demonstrated in endothelial cells, disruption of tight junction formation and stress fibre formation by depletion of $p 114$ RhoGEF, a GEF that supports junction formation, occurs in endothelial and epithelial cells ${ }^{114}$.

Similar to crosstalk with adherens junctions, crosstalk between focal adhesions and tight junctions is not limited to signalling but may also involves complex formation between focal adhesion and tight junction proteins. For example, some claudins and JAM-A have been shown to associate with integrin complexes and/or to regulate cell migration ${ }^{166-170}$, however, it is not known whether such observations reflect independent roles or is yet another example of a regulatory link between tight junctions and focal adhesions.

\section{Conclusions and perspectives}

Despite significant recent progress in structural and functional analysis of tight junctions, many open questions still remain. First of all, the topology of the adhering plasma membranes remains to be determined, and a structural model needs to be developed that explains how different junctional transmembrane proteins that have different dynamic properties can mix in the same branched intramembrane strand network and at the same time form intercellular protein complexes serving as paracellular ion-selective pores and a lipid diffusion fence. Another longstanding problem is the molecular mechanism that enables size-selective macromolecular paracellular diffusion. While the current data regarding different junctional components and their reaction to physiological stimuli would be compatible with a model based on a dynamic, remodelling strand network, the methods to visualize strand dynamics still need to be developed to validate this model. Furthermore, despite the fact that a wealth of exciting data has been generated linking particular junctional proteins to specific junctional barrier or junctional functions, it is still poorly understood how different proteins cooperate to regulate such functions, how the junctional protein network adapts to removal of specific components, and how such modified junctions respond to different physiological and pathological stimuli. Tight junction-associated signalling mechanisms have now been firmly linked to the regulation of cell proliferation, polarization and differentiation, and many of these mechanisms are evolutionarily conserved even if they might be associated with a different type of junction in different phyla. Nevertheless, we still need to establish how exactly signalling initiated at 
junctions integrates into complex signalling pathways driving diverse cellular processes. Finally, the analysis of the mechanisms by which different adhesion complexes that mediate cell-cell and cell-matrix interactions communicate and cooperate with each other will likely lead to exciting new insights into the processes that mediate epithelial and endothelial tissue development and function.

\section{Acknowledgements}

The authors are supported by the MRC, BBSRC and Fight for Sight.

\section{References}

1 Cereijido, M., Contreras, R. G. \& Shoshani, L. Cell adhesion, polarity, and epithelia in the dawn of metazoans. Physiol. Rev. 84, 1229-1262 (2004).

2 Farquhar, M. G. \& Palade, G. E. Junctional complexes in various epithelia. J. Cell Biol. 17, 375-412 (1963).

3 Claude, P. \& Goodenough, D. A. Fracture faces of zonulae occludentes from "tight" and "leaky" epithelia. J. Cell Biol. 58, 390-400 (1973).

4 Staehelin, L. A., Mukherjee, T. M. \& Williams, A. W. Freeze-etch appearance of the tight junctions in the epithelium of small and large intestine of mice. Protoplasma 67, 165-184 (1969).

5 Furuse, M. et al. Overexpression of occludin, a tight junction integral membrane protein, induces the formation of intracellular multilamellar bodies bearing tight junction-like structures. J. Cell Sci. 109, 429-435 (1996).

6 Furuse, M., Sasaki, H., Fujimoto, K. \& Tsukita, S. A single gene product, claudin-1 or -2 , reconstitutes tight junction strands and recruits occludin in fibroblasts. J. Cell Biol. 143, 391-401 (1998).

\section{Demonstration that expression of claudins is sufficient for intramembrane strand formation in} cells that lack tight junctions.

7 Morita, K., Furuse, M., Fujimoto, K. \& Tsukita, S. Claudin multigene family encoding fourtransmembrane domain protein components of tight junction strands. Proc. Natl. Acad. Sci. US A 96, 511-516 (1999).

$8 \quad$ Kubota, K. et al. $\mathrm{Ca}(2+)$-independent cell-adhesion activity of claudins, a family of integral membrane proteins localized at tight junctions. Curr. Biol. 9, 1035-1038 (1999).

9 Van Itallie, C. M. \& Anderson, J. M. Occludin confers adhesiveness when expressed in fibroblasts. J. Cell Sci. 110, 1113-1121 (1997).

10 Osler, M. E., Chang, M. S. \& Bader, D. M. Bves modulates epithelial integrity through an interaction at the tight junction. J Cell Sci 118, 4667-4678 (2005).

11 Luissint, A. C., Nusrat, A. \& Parkos, C. A. JAM-related proteins in mucosal homeostasis and inflammation. Semin. Immunopath. 36, 211-226 (2014).

12 Martin-Padura, I. et al. Junctional adhesion molecule, a novel member of the immunoglobulin superfamily that distributes at intercellular junctions and modulates monocyte transmigration. $J$. Cell Biol. 142, 117-127 (1998).

13 Cohen, C. J. et al. The coxsackievirus and adenovirus receptor is a transmembrane component of the tight junction. Proc. Natl. Acad. Sci. U S A 98, 15191-15196. (2001).

14 Higashi, T. et al. Analysis of the 'angulin' proteins LSR, ILDR1 and ILDR2--tricellulin recruitment, epithelial barrier function and implication in deafness pathogenesis. J. Cell Sci. 126, 966-977 (2013). Masuda, S. et al. LSR defines cell corners for tricellular tight junction formation in epithelial cells. $J$. Cell Sci. 124, 548-555 (2011). Lemmers, C. et al. hINADI/PATJ, a homolog of discs lost, interacts with crumbs and localizes to tight junctions in human epithelial cells. J. Biol. Chem. 277, 25408-25415 (2002). Makarova, O., Roh, M. H., Liu, C. J., Laurinec, S. \& Margolis, B. Mammalian Crumbs3 is a small transmembrane protein linked to protein associated with Lin-7 (Pals1). Gene 302, 21-29 (2003). Van Itallie, C. M. \& Anderson, J. M. Architecture of tight junctions and principles of molecular composition. Semin. Cell Dev. Biol. 36, 157-165 (2014). 
Stevenson, B. R., Siliciano, J. D., Mooseker, M. S. \& Goodenough, D. A. Identification of ZO-1: a high molecular weight polypeptide associated with the tight junction (zonula occludens) in a variety of epithelia. J. Cell Biol. 103, 755-766 (1986).

Identification of the first tight junction protein.

Rodgers, L. S., Beam, M. T., Anderson, J. M. \& Fanning, A. S. Epithelial barrier assembly requires coordinated activity of multiple domains of the tight junction protein ZO-1. J. Cell Sci. 126, 15651575 (2013).

Balda, M. S. \& Anderson, J. M. Two classes of tight junctions are revealed by ZO-1 isoforms. Am. J. Physiol. 264, C918-C924 (1993).

22 Fanning, A. S., Jameson, B. J., Jesaitis, L. A. \& Anderson, J. M. The tight junction protein ZO-1 establishes a link between the transmembrane protein occludin and the actin cytoskeleton. J. Biol. Chem. 273, 29745-29753 (1998).

Balda, M. S. \& Matter, K. The tight junction protein ZO-1 and an interacting transcription factor regulate ErbB-2 expression. EMBO J. 19, 2024-2033 (2000).

Identification of the first transcription factor regulatated by tight junctions.

Tsapara, A., Matter, K. \& Balda, M. S. The heat-shock protein Apg-2 binds to the tight junction protein ZO-1 and regulates transcriptional activity of ZONAB. Mol. Biol. Cell 17, 1322-1330 (2006). Schmidt, A. et al. Occludin binds to the SH3-hinge-GuK unit of zonula occludens protein 1: potential mechanism of tight junction regulation. Cell. Mol. Life Sci. 61, 1354-1365 (2004). Lye, M. F., Fanning, A. S., Su, Y., Anderson, J. M. \& Lavie, A. Insights into regulated ligand binding sites from the structure of ZO-1 Src homology 3-guanylate kinase module. J. Biol. Chem. 285, 13907-13917 (2010).

Fanning, A. S. et al. The unique-5 and -6 motifs of ZO-1 regulate tight junction strand localization and scaffolding properties. Mol. Biol. Cell 18, 721-731 (2007). Spadaro, D. et al. ZO proteins redundantly regulate the transcription factor DbpA/ZONAB. J. Biol. Chem. 289, 22500-22511 (2014). Gumbiner, B., Lowenkopf, T. \& Apatira, D. Identification of a 160-kDa polypeptide that binds to the tight junction protein ZO-1. Proc. Natl. Acad. Sci. U S A 88, 3460-3464 (1991). Balda, M. S., Gonzalez-Mariscal, L., Matter, K., Cereijido, M. \& Anderson, J. M. Assembly of the tight junction: the role of diacylglycerol. J. Cell Biol. 123, 293-302 (1993). Haskins, J., Gu, L., Wittchen, E. S., Hibbard, J. \& Stevenson, B. R. ZO-3, a novel member of the MAGUK protein family found at the tight junction, interacts with ZO-1 and occludin. J. Cell Biol. 141, 199-208 (1998). Ide, N. et al. Localization of membrane-associated guanylate kinase (MAGI)-1/BAI-associated protein (BAP) 1 at tight junctions of epithelial cells. Oncogene 18, 7810-7815 (1999). Dobrosotskaya, I., Guy, R. K. \& James, G. L. MAGI-1, a membrane-associated guanylate kinase with a unique arrangement of protein-protein interaction domains. J. Biol. Chem. 272, 31589-31597 (1997). Lemmers, C. et al. hINADI/PATJ, a homolog of Discs lost, interacts with crumbs and localizes to tight junctions in human epithelial cells. J. Biol. Chem. 277, 25408-25415 (2002). Roh, M. H. et al. The Maguk protein, Pals1, functions as an adapter, linking mammalian homologues of Crumbs and Discs Lost. J. Cell Biol. 157, 161-172 (2002). Hamazaki, Y., Itoh, M., Sasaki, H., Furuse, M. \& Tsukita, S. Multi-PDZ domain protein 1 (MUPP1) is concentrated at tight junctions through its possible interaction with claudin-1 and junctional adhesion molecule. J. Biol. Chem. 277, 455-461 (2002). Citi, S., Pulimeno, P. \& Paschoud, S. Cingulin, paracingulin, and PLEKHA7: signaling and cytoskeletal adaptors at the apical junctional complex. Ann. N. Y. Acad. Sci. 1257, 125-132 (2012). Yano, T., Matsui, T., Tamura, A., Uji, M. \& Tsukita, S. The association of microtubules with tight junctions is promoted by cingulin phosphorylation by AMPK. J. Cell Biol. 203, 605-614 (2013). Demonstration of a direct link between tight junctions and microtubules. tight junction proteins with distinct identities and localizations. Am. J. Physiol. 257, C621-C628 (1989). 
Cordenonsi, M. et al. Cingulin contains globular and coiled-coil domains and interacts with ZO-1, ZO-2, ZO-3, and myosin. J. Cell Biol. 147, 1569-1582 (1999).

41 Steed, E. et al. MarvelD3 couples tight junctions to the MEKK1-JNK pathway to regulate cell behavior and survival. J. Cell Biol. 204, 821-838 (2014).

Elucidation of a mechanism connecting tight junctions and JNK signalling that regulates the cellular stress response.

Fredriksson, K. et al. Proteomic analysis of proteins surrounding occludin and claudin-4 reveals their proximity to signaling and trafficking networks. PloS one 10, e0117074 (2015).

Zihni, C., Balda, M. S. \& Matter, K. Signalling at tight junctions during epithelial differentiation and microbial pathogenesis. J. Cell Sci. 127, 3401-3413 (2014).

44 Gonzalez-Mariscal, L. et al. Tight junctions and the regulation of gene expression. Semin. Cell Dev. Biol. 36, 213-223 (2014).

45 Quiros, M. \& Nusrat, A. RhoGTPases, actomyosin signaling and regulation of the epithelial Apical Junctional Complex. Semin. Cell Dev. Biol. 36, 194-203 (2014).

Adachi, M. et al. Normal establishment of epithelial tight junctions in mice and cultured cells lacking expression of ZO-3, a tight-junction MAGUK protein. Mol. Cell. Biol. 26, 9003-9015 (2006). Guillemot, L. et al. Cingulin is dispensable for epithelial barrier function and tight junction structure, and plays a role in the control of claudin-2 expression and response to duodenal mucosa injury. J. Cell Sci. (2012).

Xu, J. et al. Early embryonic lethality of mice lacking ZO-2, but Not ZO-3, reveals critical and nonredundant roles for individual zonula occludens proteins in mammalian development. Mol. Cell. Biol. 28, 1669-1678 (2008).

Katsuno, T. et al. Deficiency of ZO-1 Causes Embryonic Lethal Phenotype Associated with Defected Yolk Sac Angiogenesis and Apoptosis of Embryonic Cells. Mol. Biol. Cell (2008).

Tokuda, S., Higashi, T. \& Furuse, M. ZO-1 knockout by TALEN-mediated gene targeting in MDCK cells: involvement of ZO-1 in the regulation of cytoskeleton and cell shape. PloS one 9, e104994 (2014).

Kiener, T. K., Selptsova-Friedrich, I. \& Hunziker, W. Tjp3/zo-3 is critical for epidermal barrier function in zebrafish embryos. Dev. Biol. 316, 36-49 (2008).

52 Mir, H. et al. Occludin deficiency promotes ethanol-induced disruption of colonic epithelial junctions, gut barrier dysfunction and liver damage in mice. Biochim. Biophys. Acta (2015).

References $\mathbf{4 8}$ to $\mathbf{5 2}$ provide striking examples of specific physiological roles for tight junction proteins that are often erroneoulsy considered as being redundant or not critical for barrier function.

53 Suzuki, H. et al. Crystal structure of a claudin provides insight into the architecture of tight junctions. Science 344, 304-307 (2014).

Determination of the strucutre of a claudin enabling more detailed modelling of the structure of tight junctions and the possible route of ion permeation.

54 Suzuki, H., Tani, K., Tamura, A., Tsukita, S. \& Fujiyoshi, Y. Model for the architecture of claudinbased paracellular ion channels through tight junctions. J. Mol. Biol. 427, 291-297 (2015).

Furuse, M., Sasaki, H. \& Tsukita, S. Manner of interaction of heterogeneous claudin species within and between tight junction strands. J. Cell Biol. 147, 891-903 (1999).

Tsukita, S. \& Furuse, M. Occludin and claudins in tight-junction strands: leading or supporting players? Trends Cell Biol. 9, 268-273 (1999).

57 Haseloff, R. F., Dithmer, S., Winkler, L., Wolburg, H. \& Blasig, I. E. Transmembrane proteins of the tight junctions at the blood-brain barrier: structural and functional aspects. Semin. Cell Dev. Biol. 38, 16-25 (2015).

Shen, L., Weber, C. R. \& Turner, J. R. The tight junction protein complex undergoes rapid and continuous molecular remodeling at steady state. J. Cell Biol. 181, 683-695 (2008).

Demonstration that tight junctions are not a rigid complex and that different junctional proteins have distinct dynamic properties 
Chernomordik, L. V. \& Kozlov, M. M. Membrane hemifusion: crossing a chasm in two leaps. Cell 123, 375-382 (2005).

61 Kachar, B. \& Reese, T. S. Evidence for the lipidic nature of tight junction strands. Nature 296, 464466 (1982).

62 Kan, F. W. Cytochemical evidence for the presence of phospholipids in epithelial tight junction strands. J. Histochem. Cytochem. 41, 649-656 (1993).

Fujimoto, K. Freeze-fracture replica electron microscopy combined with SDS digestion for cytochemical labeling of intergral membrane proteins: aplication to the immunogold labeling of intercellular junctional complex. J. Cell Sci. 108, 3443-3449 (1995).

Cording, J. et al. In tight junctions, claudins regulate the interactions between occludin, tricellulin and marvelD3, which, inversely, modulate claudin oligomerization. J Cell Sci 126, 554-564 (2013). van Meer, G., Gumbiner, B. \& Simons, K. The tight junction does not allow lipid molecules to diffuse from one epithelial cell to the next. Nature 322, 639-641 (1986).

Grebenkamper, K. \& Galla, H. J. Translational diffusion measurements of a fluorescent phospholipid between MDCK-I cells support the lipid model of the tight junctions. Chem. Phys. Lipids 71, 133-143 (1994).

Nusrat, A. et al. Tight junctions are membrane microdomains. J. Cell Sci. 113 ( Pt 10), 1771-1781 (2000). function by altering the detergent solubility and distribution of specific tight-junction proteins. Biochem. J. 387, 553-560 (2005). Lynch, R. D. et al. Cholesterol depletion alters detergent-specific solubility profiles of selected tight junction proteins and the phosphorylation of occludin. Exp. Cell Res. 313, 2597-2610 (2007). Calderon, V. et al. Tight junctions and the experimental modifications of lipid content. J. Membr. Biol. 164, 59-69 (1998).

Francis, S. A. et al. Rapid reduction of MDCK cell cholesterol by methyl-beta-cyclodextrin alters steady state transepithelial electrical resistance. Eur. J. Cell Biol. 78, 473-484 (1999). Larre, I., Ponce, A., Franco, M. \& Cereijido, M. The emergence of the concept of tight junctions and physiological regulation by ouabain. Semin. Cell Dev. Biol. 36, 149-156 (2014). Yu, A. S. Claudins and the kidney. J. Am. Soc. Nephrol. 26, 11-19 (2015). Yu, A. S. et al. Molecular basis for cation selectivity in claudin-2-based paracellular pores: identification of an electrostatic interaction site. J. Gen. Pphysiol. 133, 111-127 (2009). Lingaraju, A., Long, T. M., Wang, Y., Austin, J. R., 2nd \& Turner, J. R. Conceptual barriers to understanding physical barriers. Semin. Cell Dev. Biol. 42, 13-21 (2015). Steed, E., Balda, M. S. \& Matter, K. Dynamics and functions of tight junctions. Trends Cell Biol. 20, 142-149 (2010).

77 Simon, D. B. et al. Paracellin-1, a renal tight junction protein required for paracellular $\mathrm{Mg} 2+$ resorption. Science 285, 103-106 (1999).

Identification of a tight junction component required for ion-specific paracellular diffusion. McCarthy, K. M. et al. Inducible expression of claudin-1-myc but not occludin-VSV-G results in aberrant tight junction strand formation in MDCK cells. J. Cell Sci. 113, 3387-3398 (2000). Furuse, M. et al. Claudin-based tight junctions are crucial for the mammalian epidermal barrier: a lesson from claudin-1-deficient mice. J. Cell Biol. 156, 1099-1111. (2002). Amasheh, S. et al. Claudin-2 expression induces cation-selective channels in tight junctions of epithelial cells. J. Cell Sci. 115, 4969-4976 (2002). Furuse, M., Furuse, K., Sasaki, H. \& Tsukita, S. Conversion of zonulae occludentes from tight to leaky strand type by introducing claudin-2 into Madin-Darby canine kidney I cells. J. Cell Biol. 153, 263272. (2001).

\section{Demonstration that the claudin composition of a junction determines transepithelial electrical} resistance. 

localization and function. J. Cell Sci. 122, 1507-1517 (2009). Krug, S. M. et al. Claudin-17 forms tight junction channels with distinct anion selectivity. Cell. Mol. Life Sci. 69, 2765-2778 (2012).

Krug, S. M., Schulzke, J. D. \& Fromm, M. Tight junction, selective permeability, and related diseases. Semin. Cell Dev. Biol. 36, 166-176 (2014). selective channels in the paracellular pathway between epithelial cells. Am. J. Physiol. Cell Physiol. 283, C142-147 (2002).

\section{Establishes that the claudin repertoire of a cell is important for the ion selectivity of the} paracellualr pathway and provides evidence for the roles of the extracellular domains.

Angelow, S. \& Yu, A. S. Structure-function studies of claudin extracellular domains by cysteinescanning mutagenesis. J. Biol. Chem. 284, 29205-29217 (2009). Gunzel, D. \& Yu, A. S. Claudins and the modulation of tight junction permeability. Physiol. Rev. 93, 525-569 (2013).

Weber, C. R. et al. Claudin-2-dependent paracellular channels are dynamically gated. Elife 4 (2015). Demonstration of paracellular claudin pores using patch clamp.

Tamura, A. et al. Loss of claudin-15, but not claudin-2, causes $\mathrm{Na}+$ deficiency and glucose malabsorption in mouse small intestine. Gastroenterology 140, 913-923 (2011). Wada, M., Tamura, A., Takahashi, N. \& Tsukita, S. Loss of claudins 2 and 15 from mice causes defects in paracellular $\mathrm{Na}+$ flow and nutrient transport in gut and leads to death from malnutrition. Gastroenterology 144, 369-380 (2013).

Wilcox, E. R. et al. Mutations in the gene encoding tight junction claudin-14 cause autosomal recessive deafness DFNB29. Cell 104, 165-172 (2001).

Wen, H., Watry, D. D., Marcondes, M. C. \& Fox, H. S. Selective decrease in paracellular conductance of tight junctions: role of the first extracellular domain of claudin-5. Mol. Cell. Biol. 24, 8408-8417 (2004). relationships based on homology models and crystal structures. Semin. Cell Dev. Biol. 42, 3-12 (2015). physiology and hypertension. Proc. Natl. Acad. Sci. U S A 101, 14877-14882 (2004). Wilson, F. H. et al. Human hypertension caused by mutations in WNK kinases. Science 293, 11071112 (2001).

97 Yamauchi, K. et al. Disease-causing mutant WNK4 increases paracellular chloride permeability and phosphorylates claudins. Proc. Natl. Acad. Sci. U S A 101, 4690-4694 (2004).

98 Ohta, A. et al. Overexpression of human WNK1 increases paracellular chloride permeability and phosphorylation of claudin-4 in MDCKII cells. Biochem. Biophys. Res. Commun. 349, 804-808 (2006). Tatum, R. et al. WNK4 phosphorylates ser(206) of claudin-7 and promotes paracellular $\mathrm{Cl}(-)$ permeability. FEBS Lett. 581, 3887-3891 (2007).

100 Diamond, J. M. Twenty-first Bowditch lecture. The epithelial junction: bridge, gate, and fence. Physiologist 20, 10-18 (1977).

101 van Meer, G. \& Simons, K. The function of tight junctions in maintaining differences in lipid composition between the apical and the basolateral cell surface domains of MDCK cells. EMBO J. 5 , 1455-1464 (1986).

102 Mandel, L. J., Bacallao, R. \& Zampighi, G. Uncoupling of the molecular 'fence' and paracellular 'gate' functions in epithelial tight junctions. Nature 361, 552-555 (1993).

103 Nava, P., Lopez, S., Arias, C. F., Islas, S. \& Gonzalez-Mariscal, L. The rotavirus surface protein VP8 modulates the gate and fence function of tight junctions in epithelial cells. J. Cell Sci. 117, 55095519 (2004).

104 Balda, M. S. et al. Functional dissociation of paracellular permeability and transepithelial electrical resistance and disruption of the apical-basolateral intramembrane diffusion barrier by expression of a mutant tight junction membrane protein. J. Cell Biol. 134, 1031-1049 (1996). 
105 Umeda, K. et al. ZO-1 and ZO-2 independently determine where claudins are polymerized in tightjunction strand formation. Cell 126, 741-754 (2006).

106 Ikenouchi, J. et al. Lipid polarity is maintained in absence of tight junctions. J. Biol. Chem. 287, 95259533 (2012).

107 Cereijido, M., Robbins, E. S., Dolan, W. J., Rotunno, C. A. \& Sabatini, D. D. Polarized monolayers formed by epithelial cells on a permeable and translucent support. J. Cell Biol. 77, 853-880 (1978). Establishes the today commonly used model of culturing epithelial cells on a permeable support.

108 Balda, M. S. et al. Assembly and sealing of tight junctions: possible participation of G-proteins, phospholipase C, protein kinase C and calmodulin. J. Mem. Biol. 122, 193-202 (1991).

109 Rajasekaran, A. K., Hojo, M., Huima, T. \& Rodriguez-Boulan, E. Catenins and zonula occludens-1 form a complex during early stages in the assembly of tight junctions. J. Cell Biol. 132, 451-463 (1996).

110 Maiers, J. L., Peng, X., Fanning, A. S. \& DeMali, K. A. ZO-1 recruitment to alpha-catenin--a novel mechanism for coupling the assembly of tight junctions to adherens junctions. J. Cell Sci. 126, 39043915 (2013).

111 Fukuhara, A. et al. Involvement of nectin in the localization of junctional adhesion molecule at tight junctions. Oncogene 21, 7642-7655 (2002).

112 Garrido-Urbani, S., Bradfield, P. F. \& Imhof, B. A. Tight junction dynamics: the role of junctional adhesion molecules (JAMs). Cell Tissue Res. 355, 701-715 (2014).

113 Herrmann, J. R. \& Turner, J. R. Beyond Ussing's chambers: contemporary thoughts on integration of transepithelial transport. Am. J. Physiol. Cell Physiol. 310, C423-431 (2016).

114 Terry, S. J. et al. Spatially restricted activation of RhoA signalling at epithelial junctions by p114RhoGEF drives junction formation and morphogenesis. Nat. Cell Biol. 13, 159-166 (2011). Identification of a molecular mechanisms that mediates tight junction-specific RhoA and myosin activation and, thereby, formation of functional barriers.

115 Itoh, M., Tsukita, S., Yamazaki, Y. \& Sugimoto, H. Rho GTP exchange factor ARHGEF11 regulates the integrity of epithelial junctions by connecting ZO-1 and RhoA-myosin II signaling. Proc. Natl. Acad. Sci. USA 109, 9905-9910 (2012).

116 Tornavaca, O. et al. ZO-1 controls endothelial adherens junctions, cell-cell tension, angiogenesis, and barrier formation. J. Cell Biol. 208, 821-838 (2015).

Demonstration that tight junctions regulate tension on adherens junctions and angiogenesis

117 Otani, T., Ichii, T., Aono, S. \& Takeichi, M. Cdc42 GEF Tuba regulates the junctional configuration of simple epithelial cells. J. Cell Biol. 175, 135-146 (2006).

118 Oda, Y., Otani, T., Ikenouchi, J. \& Furuse, M. Tricellulin regulates junctional tension of epithelial cells at tricellular contacts through Cdc42. J. Cell Sci. 127, 4201-4212 (2014).

Determination of a regualtory function of tricellulin for $\mathrm{Cdc} 42$ and control of tensin between tricellular corners

119 Schluter, M. A. \& Margolis, B. Apicobasal polarity in the kidney. Exp. Cell Res. 318, 1033-1039 (2012).

120 Itoh, M. et al. Junctional adhesion molecule (JAM) binds to PAR-3: a possible mechanism for the recruitment of PAR-3 to tight junctions. J. Cell Biol. 154, 491-497 (2001).

121 Ebnet, K., Iden, S., Gerke, V. \& Suzuki, A. Regulation of epithelial and endothelial junctions by PAR proteins. Front. Biosci. 13, 6520-6536 (2008).

122 Liu, X. F., Ishida, H., Raziuddin, R. \& Miki, T. Nucleotide exchange factor ECT2 interacts with the polarity protein complex Par6/Par3/protein kinase Czeta (PKCzeta) and regulates PKCzeta activity. Mol. Cell Biol. 24, 6665-6675 (2004).

123 Wells, C. D. et al. A Rich1/Amot complex regulates the Cdc42 GTPase and apical-polarity proteins in epithelial cells. Cell 125, 535-548 (2006).

124 Elbediwy, A. et al. Epithelial junction formation requires confinement of $\mathrm{Cdc} 42$ activity by a novel SH3BP1 complex. J. Cell Biol. 198, 677-693 (2012).

125 Zihni, C. et al. Dbl3 drives Cdc42 signaling at the apical margin to regulate junction position and apical differentiation. J. Cell Biol. 204, 111-127 (2014).

Elucidation of the molecular mechanism that drives polarized Cdc42 activation at the apical pole. 
126 Morais-de-Sa, E., Mirouse, V. \& St Johnston, D. aPKC phosphorylation of Bazooka defines the apical/lateral border in Drosophila epithelial cells. Cell 141, 509-523 (2010).

127 Walther, R. F. \& Pichaud, F. Crumbs/DaPKC-dependent apical exclusion of Bazooka promotes photoreceptor polarity remodeling. Curr. Biol. 20, 1065-1074 (2010).

128 Michel, D. et al. PATJ connects and stabilizes apical and lateral components of tight junctions in human intestinal cells. J. Cell Sci. 118, 4049-4057 (2005).

129 Adachi, M. et al. Similar and distinct properties of MUPP1 and Patj, two homologous PDZ domaincontaining tight-junction proteins. Mol. Cell. Biol. 29, 2372-2389 (2009).

130 Roh, M. H., Liu, C. J., Laurinec, S. \& Margolis, B. The carboxyl terminus of zona occludens-3 binds and recruits a mammalian homologue of discs lost to tight junctions. J. Biol. Chem. 277, 2750127509 (2002).

131 Lemmers, C. et al. CRB3 binds directly to Par6 and regulates the morphogenesis of the tight junctions in mammalian epithelial cells. Mol. Biol. Cell 15, 1324-1333 (2004).

132 Ellenbroek, S. I., Iden, S. \& Collard, J. G. Cell polarity proteins and cancer. Semin. Cancer Biol. 22, 208-215 (2012).

133 Zhao, B. et al. Angiomotin is a novel Hippo pathway component that inhibits YAP oncoprotein. Genes Dev. 25, 51-63 (2011).

134 Oka, T. et al. Functional complexes between YAP2 and ZO-2 are PDZ domain-dependent, and regulate YAP2 nuclear localization and signalling. Biochem. J. 432, 461-472 (2010).

135 Cravo, A. S. et al. Hippo pathway elements Co-localize with Occludin: A possible sensor system in pancreatic epithelial cells. Tissue Barr. 3, e1037948 (2015).

$136 \mathrm{Lv}, \mathrm{X}$. B. et al. PARD3 induces TAZ activation and cell growth by promoting LATS1 and PP1 interaction. EMBO Rep. 16, 975-985 (2015).

$137 \mathrm{Yi}, \mathrm{C}$. et al. A tight junction-associated Merlin-angiomotin complex mediates Merlin's regulation of mitogenic signaling and tumor suppressive functions. Cancer Cell 19, 527-540 (2011).

138 Zhang, N. et al. The Merlin/NF2 tumor suppressor functions through the YAP oncoprotein to regulate tissue homeostasis in mammals. Dev. Cell 19, 27-38 (2010).

139 Balda, M. S., Garrett, M. D. \& Matter, K. The ZO-1-associated Y-box factor ZONAB regulates epithelial cell proliferation and cell density. J. Cell Biol. 160, 423-432 (2003).

140 Nie, M., Aijaz, S., Leefa Chong San, I. V., Balda, M. S. \& Matter, K. The Y-box factor ZONAB/DbpA associates with GEF-H1/LfC and mediates Rho-stimulated transcription. EMBO Rep. 10, 1125-1131 (2009).

141 Kavanagh, E. et al. Functional interaction between the ZO-1-interacting transcription factor ZONAB/DbpA and the RNA processing factor symplekin. J. Cell Sci. 119, 5098-5105 (2006).

142 Frankel, P. et al. RalA interacts with ZONAB in a cell density-dependent manner and regulates its transcriptional activity. EMBO J. 24, 54-62 (2005).

143 Ikari, A. et al. Nuclear distribution of claudin-2 increases cell proliferation in human lung adenocarcinoma cells. Biochim. Biophys. Acta 1843, 2079-2088 (2014).

144 Pannequin, J. et al. Phosphatidylethanol accumulation promotes intestinal hyperplasia by inducing ZONAB-mediated cell density increase in response to chronic ethanol exposure. Mol. Can. Res. 5, 1147-1157 (2007).

145 Buchert, M. et al. Symplekin promotes tumorigenicity by up-regulating claudin-2 expression. Proc. Natl. Acad. Sci. USA 107, 2628-2633 (2010).

146 Sourisseau, T. et al. Regulation of PCNA and cyclin D1 expression and epithelial morphogenesis by the ZO-1-regulated transcription factor ZONAB/DbpA. Mol. Cell Biol. 26, 2387-2398 (2006).

147 Ruan, Y. C. et al. CFTR interacts with ZO-1 to regulate tight junction assembly and epithelial differentiation through the ZONAB pathway. J. Cell Sci. 127, 4396-4408 (2014).

148 Russ, P. K. et al. Bves modulates tight junction associated signaling. PloS one 6, e14563 (2011).

149 Liu, L. B. et al. Bradykinin increased the permeability of BTB via NOS/NO/ZONAB-mediating downregulation of claudin-5 and occludin. Biochem. Biophys. Res. Commun. 464, 118-125 (2015).

150 Dominguez-Calderon, A. et al. ZO-2 silencing induces renal hypertrophy through a cell cycle mechanism and the activation of YAP and the mTOR pathway. Mol. Biol. Cell (2016). 
151 Li, D. \& Mrsny, R. J. Oncogenic Raf-1 Disrupts Epithelial Tight Junctions via Downregulation of Occludin. J. Cell Biol. 148, 791-800 (2000).

152 Nusrat, A. et al. The coiled-coil domain of occludin can act to organize structural and functional elements of the epithelial tight junction. J. Biol. Chem. 275, 29816-29822 (2000).

153 Barrios-Rodiles, M. et al. High-throughput mapping of a dynamic signaling network in mammalian cells. Science 307, 1621-1625 (2005).

154 Lockwood, C., Zaidel-Bar, R. \& Hardin, J. The C. elegans zonula occludens ortholog cooperates with the cadherin complex to recruit actin during morphogenesis. Curr. Biol. 18, 1333-1337 (2008).

155 Nie, M., Balda, M. S. \& Matter, K. Stress- and Rho-activated ZO-1-associated nucleic acid binding protein binding to p21 mRNA mediates stabilization, translation, and cell survival. Proc. Natl. Acad. Sci. USA 109, 10897-10902 (2012).

156 Monteiro, A. C. et al. Trans-dimerization of JAM-A regulates Rap2 and is mediated by a domain that is distinct from the cis-dimerization interface. Mol. Biol. Cell 25, 1574-1585 (2014).

157 Severson, E. A., Lee, W. Y., Capaldo, C. T., Nusrat, A. \& Parkos, C. A. Junctional adhesion molecule A interacts with Afadin and PDZ-GEF2 to activate Rap1A, regulate beta1 integrin levels, and enhance cell migration. Mol. Biol. Cell 20, 1916-1925 (2009).

158 Cera, M. R. et al. JAM-A promotes neutrophil chemotaxis by controlling integrin internalization and recycling. J. Cell Sci. 122, 268-277 (2009).

159 McSherry, E. A., Brennan, K., Hudson, L., Hill, A. D. K. \& Hopkins, A. M. Breast cancer cell migration is regulated through junctional adhesion molecule-A-mediated activation of Rap1 GTPase. Breast Cancer Res. 13 (2011).

References 156-159 establish JAM-A as a regulator of focal adhesions and adherens junctions via Rap GTPases.

160 Kuo, J. C., Han, X., Hsiao, C. T., Yates, J. R., 3rd \& Waterman, C. M. Analysis of the myosin-IIresponsive focal adhesion proteome reveals a role for beta-Pix in negative regulation of focal adhesion maturation. Nat. Cell Biol. 13, 383-393 (2011).

161 Ren, Y., Li, R., Zheng, Y. \& Busch, H. Cloning and characterization of GEF-H1, a microtubuleassociated guanine nucleotide exchange factor for Rac and Rho GTPases. J. Biol. Chem. 273, 3495434960 (1998).

162 Krendel, M., Zenke, F. T. \& Bokoch, G. M. Nucleotide exchange factor GEF-H1 mediates cross-talk between microtubules and the actin cytoskeleton. Nat. Cell Biol. 4, 294-301 (2002).

163 Aijaz, S., D'Atri, F., Citi, S., Balda, M. S. \& Matter, K. Binding of GEF-H1 to the tight junctionassociated adaptor cingulin results in inhibition of Rho signaling and G1/S phase transition. Dev. Cell 8, 777-786 (2005).

Identification of a mechanism by which tight junctions contribute to downregulation of cellular RhoA signalling upon formation of mature monolayers.

164 Huang, I. H. et al. GEF-H1 controls focal adhesion signaling that regulates mesenchymal stem cell lineage commitment. J. Cell Sci. 127, 4186-4200 (2014).

165 Guilluy, C. et al. The Rho GEFs LARG and GEF-H1 regulate the mechanical response to force on integrins. Nat. Cell Biol. 13, 722-727 (2011).

166 Tiwari-Woodruff, S. K. et al. OSP/claudin-11 forms a complex with a novel member of the tetraspanin super family and beta1 integrin and regulates proliferation and migration of oligodendrocytes. J. Cell Biol. 153, 295-305. (2001).

167 Lu, Z. et al. A non-tight junction function of claudin-7-Interaction with integrin signaling in suppressing lung cancer cell proliferation and detachment. Mol. Can. 14, 120 (2015).

168 Dhawan, P. et al. Claudin-1 regulates cellular transformation and metastatic behavior in colon cancer. J. Clin. Invest. 115, 1765-1776 (2005).

169 Peddibhotla, S. S. et al. Tetraspanin CD9 links junctional adhesion molecule-A to alphavbeta3 integrin to mediate basic fibroblast growth factor-specific angiogenic signaling. Mol. Biol. Cell 24, 933-944 (2013).

170 Naik, M. U. \& Naik, U. P. Junctional adhesion molecule-A-induced endothelial cell migration on vitronectin is integrin alpha v beta 3 specific. J. Cell Sci. 119, 490-499 (2006). 
171 Izumi, Y. \& Furuse, M. Molecular organization and function of invertebrate occluding junctions. Semin. Cell Dev. Biol. 36, 186-193 (2014).

172 Simske, J. S. \& Hardin, J. Claudin family proteins in Caenorhabditis elegans. Methods Mol. Biol. 762, 147-169 (2011).

173 Wu, V. M., Schulte, J., Hirschi, A., Tepass, U. \& Beitel, G. J. Sinuous is a Drosophila claudin required for septate junction organization and epithelial tube size control. J. Cell Biol. 164, 313-323 (2004).

$174 \mathrm{Wu}, \mathrm{V}$. M. et al. Drosophila Varicose, a member of a new subgroup of basolateral MAGUKs, is required for septate junctions and tracheal morphogenesis. Develop. 134, 999-1009 (2007).

175 Behr, M., Riedel, D. \& Schuh, R. The claudin-like megatrachea is essential in septate junctions for the epithelial barrier function in Drosophila. Dev. Cell 5, 611-620 (2003).

176 Genova, J. L. \& Fehon, R. G. Neuroglian, Gliotactin, and the Na+/K+ ATPase are essential for septate junction function in Drosophila. J. Cell Biol. 161, 979-989 (2003).

177 Nelson, K. S., Furuse, M. \& Beitel, G. J. The Drosophila Claudin Kune-kune is required for septate junction organization and tracheal tube size control. Genetics 185, 831-839 (2010).

178 Asano, A., Asano, K., Sasaki, H., Furuse, M. \& Tsukita, S. Claudins in Caenorhabditis elegans: their distribution and barrier function in the epithelium. Curr. Biol. 13, 1042-1046 (2003).

179 Suzuki, A. \& Ohno, S. The PAR-aPKC system: lessons in polarity. J. Cell Sci. 119, 979-987 (2006).

180 Matter, K. \& Balda, M. S. Signalling to and from tight junctions. Nat. Rev. Mol. Cell Biol. 4, 225-236 (2003). 


\section{Author biographies \\ Ceniz Zihni}

After obtaining his Ph.D. degree from the University of London, Ceniz Zihni continued his study of the role of RhoGTPases and the cytoskeleton in cellular processes that underlie tissue homeostasis, including cellular proliferation/apoptosis control. He is currently working as a senior postdoctoral fellow in the laboratories of Karl Matter and Maria S Balda at University College London, Institute of Ophthalmology, where he has made fundamental, key discoveries of how molecular mechanisms integrate RhoGTPase-signalling with cytoskeletal reorganization to control and coordinate epithelial proliferation, polarization and differentiation. He also has a keen interest in how these processes may be dysregulated in various diseases including cancer and age-related degeneration of the eye.

\section{Clare Mills}

Clare Mills trained as a Ph.D. student with Maria S. Balda and Karl Matter at University College London, where she investigated pathological RhoA signalling in endothelial and epithelial cells to develop novel therapeutic approaches. Currently, she is a scientist at Randox Laboratories, Crumlin, UK.

\section{Karl Matter}

Karl Matter obtained his Ph.D. from the University of Basel, Switzerland. After postdoctoral training at Yale University, USA, he moved to the University of Geneva, Switzerland, as a group leader. Since 2001, he is at the Institute of Ophthalmology, University College London, UK, where he is a Professor of Cell Biology. His laboratory is interested in tight junctions and mechanisms regulating cell polarity.

\section{Maria S. Balda}

Maria S. Balda trained in Buenos Aires, Argentina, obtained her Ph.D. from the Centre for Research and Advanced Studies in Mexico, Mexico City, and received postdoctoral training at Yale University, USA. She moved then to the University of Geneva, Switzerland, and, in 2001, to the Institute of Ophthalmology, University College London, UK, where she is a Professor of Cell Biology. Her laboratory is interested in endothelial and epithelial cell adhesion, tight junctions and signal transduction.

\section{Online summary}

- Tight junctions are intercellular adhesion complex in epithelia and endothelia that control paracellular permeability. This paracellular diffusion barrier is semipermeable as it is size- and charge-selective.

- Paracellular ion permeability is largely determined by the claudin composition of tight junctions, a family of transmembrane proteins that are thought to form gated ion-selective paracellular pores through the paracellular diffusion barrier.

- Tight junctions form the border between the apical and basolateral cell surface domains in polarized epithelia, and support the maintenance of cell surface polarity by restricting intermixing of apical and basolateral transmembrane components. 
- Tight junctions are an integral component of the evolutionarily conserved signalling mechanisms that control epithelial cell polarization and the formation of morphologically and functionally distinct apical domains.

- Tight junctions form bidirectional signalling platforms that receive signals from the cell interior regulate their assembly and function, and that transduce signals to the cell interior to control cell proliferation, migration, differentiation and survival.

- Tight junctions are part of an interconnected network of adhesion complexes that includes adherens junctions and focal adhesions. These adhesion complexes crosstalk via direct proteinprotein interactions as well as by transmitting signals to each other that influence their assembly and function.

\section{Glossary}

Actomyosin - cytoskeletal fibres formed by F-actin and myosin II.

Desmosomes - an adhesive structure, also known as maculae adherents, formed from dense protein plaques of two adjacent cells, with associated intermediate filaments and transmembrane proteins known as cadherins.

Immunoglobulin-like domain - a protein domain consisting of a double layer sandwich of 7-9 antiparallel $\beta$-stands arranged in two $\beta$-sheets.

Osmoregulation - a process utilized by cells and simple organisms to maintain fluid and electrolyte balance with their immediate environment.

Lipid Micelles - lipid molecules arranged in a spherical form in aqueaous solutions in response to the amphipathic nature of fatty acids, i.e. containing both a hydrophilic, polar head group and a long hydrophilic chain.

Patch clamp approach - an electrophysiology technique that allows the study of single and multiple ion channels in membranes.

Homology Model - a comparative modelling of a protein through construction of an atomic resolution model of the 'test' protein from its amino acid sequence and a resolved threedimensional structure of a related homologous protein 'used' as a template.

Guanine nucleotide exchange factor (GEF) - proteins that activate monomeric GTPases by stimulating the dissociation of guanosine diphosphate (GDP), thereby permitting binding of guanosine triphosphate (GTP).

Small GTPase - small monomeric proteins homologous to Ras that exist in an inactive GDP-bound form and an active GTP-bound form in which they activate other signalling proteins.

Heterotrimeric GTPase - also called G-proteins and consist of three subunits: the GTP-binding $\alpha$ subunit and the smaller $\beta$ and $y$ subunits that have regulatory and signalling functions.

Hyperosmotic stress - a phenomenon experienced by cells and tissues when extracellular fluid osmolarity exceeds that of the intracellular fluid.

Focal Adhesions - large dynamic protein complexes that link the cytoskeleton of a cell to the extracellular matrix (ECM). 
Stress fibres - contractile actin bundles in non-muscle cells comprising of actin microfilaments, myosin II and crosslinkers such as a-actinin.

MARVEL Domain - a four transmembrane helix module that has been identified in proteins of various families, many of which associated with cholesterol-rich membrane microdomains. 


\section{Figure Legends}

Figure 1. The junctional complex and tight junctions.

(A) The junctional complex in epithelial and endothelial cells. Tight junctions (orange) are apically located in polarised epithelial cells and often intermixed with adherens junctions (green) in endothelial cells. (B) Brief overview of the types of tight junction proteins: transmembrane and cytoplasmic plaque proteins. Indicted are only representatives of the main groups of tight junction proteins: transmembrane proteins (Crb3; Marvel domain proteins like occludin, tricellulin, and MarvelD3; claudins; JAMs; BVES), adaptor proteins and cytoskeletal linkers (zonula occludens ZO proteins, cingulin, Pals-1, PATJ, MAGI, PAR3 and PAR6), and signalling components (aPKC; the small RhoGTPases Cdc42, Rac and RhoA; and guanine nucleotide exchange factors for Rho GTPases). Indicated are also the adherens junction complexes based on E-cadherin and nectin and their main cytosolic interaction partners (E-cadherin: p120catenin, $\alpha$ - and $\beta$-catenin; nectins: AF-6) that are mentioned in this review. (C) Super-resolution immunofluorescence image of the tight junction cytoplasmic plaque proteins ZO-1 and cingulin illustrating the apparently ordered, regular structure of the junctional plaque (shown is an image of renal epithelial cells obtained with a gated stimulated emission depletion microscope. (D) Scheme of areas of apparent hemifusions of neighbouring cells where tight junction strands are located. (E) A freeze fracture electron microscopy image of the tight junction strand network along the apical membrane domain of intestinal epithelial cells (Image, Peter Munro).

\section{Figure 2. Tight Junctions structure and function working models.}

(A) Models of tight junction. The protein model relies on intercellular protein-protein interactions for the formation of a paracellular diffusion barrier between two plasma membranes formed by standard lipid bilayers. In the lipid-protein hybrid model, the continuity of the lipid bilayer is interrupted by cylinder-shaped inverted micelles that allow areas of hemifusions of the two neighbouring plasma membranes that are stabilised by transmembrane proteins. In such a model, the exoplasmic leaflets of neighbouring cells are continuous. (B) The fence and gate functions. Integral transmembrane protein components are shown to act as a fence for diffusion of lipids along the exoplasmic leaflet; in a hybrid model, the contact site would also contain inverted micelles. Indicated is also the gate function, which refers to a regulated semipermeable diffusion barrier that controls diffusion along the paracellular space. (C, D) Specificity of the paracellular gate and mechanisms of diffusion. The paracellular diffusion barrier is semipermeable and differentiates solutes on the basis of size and charge. Size-selective macromolecular diffusion of tracers (green particles, hydrophilic molecules that can diffuse across the junction; red particles, molecules too large to cross tight junctions) and ion conductance are though to be mediated by two distinct mechanisms. Ion conductance is mediated by gated channels that can be open or closed and are ion-selective. These channels are formed by intercellular claudin complexes forming a pore-like structure (see figure 4). Size-selective macromolecular tracer diffusion is less well understood but may involve dynamic properties of the intramembrane strands such as remodelling of the branches or even dissociation/reformation of strand sections, leading to transient openings to allow the stepwise diffusion across the junction (depicted in panel $D$, which represents a schematic en face view of a section through tight junctions along the contacts between two neighbouring cells). The indicated serial diffusion barriers are thought to be represented by the intramembrane strands seen in freeze fracture replicas.

\section{Figure 3. Structure of claudins and intercellular pore formation.}

(A) Scheme of claudin structure and motifs. The crystal structure of claudin-15 has revealed a characteristic $\beta$-sheet fold of the two extracellular domains that is anchored to a transmembrane four-helix bundle (TM 1-4). The two extracellular domains or 'loops' (ECL1 and ECL2) are 
important for ion-selectivity of the paracellular pathway due to specific correspondingly charged residues (e.g., claudin-15 has negatively charged residues and forms a cation-selective pore). (B, C) Claudins are thought to dimerise face-to-face through interactions between the edges of the extracellular $\beta$-sheets as well as to interact with neighbouring claudin molecules within the same plasma membrane (here annotated as protein 1 (P1) and protein 2 (P2)). Three of the transmembrane domains (TM1, 2 and 4; orange) have the exact length required to span a lipid bilayer; the third transmembrane domain is longer (blue) and it is thought that the extended hydrophobic domain is important for the interaction with the adjacent protomer. The two indicated cysteins in the extracellular loop 1 (ECL1) form a disulphide bond that is structurally essential. V1 and $\mathrm{V} 2$ refer to variable, flexible regions that may be important for the specificity of interactions between claudins of neighbouring cells (cell-cell interaction). (B, C) The interactions between claudin molecules in cis (i.e., within the same membrane) and in trans (i.e., with molecules in the neighbouring membrane) is thought to result in the formation of two anti-parallel claudin polymers in each membrane and are proposed to represent the intramembrane strands seen in freeze facture replicas. The two sets of anti-parallel strands form intercellular adhesions by face-to-face interactions of claudin molecules, protomers, resulting in the formation of paracellular pores. These paracellular pores are gated (i.e., they can be either opened or closed); however, the structural changes underlying gating are not known.

\section{Figure 4. Junction assembly and crosstalk between adhesion complexes.}

(A) Epithelial cells form cell-cell junctions by assembling a primordial junction initiated by Ecadherin and nectin, leading to the recruitment of tight junction components due to interactions between bona fide tight and adherens junction components such as ZO-1 and a-catenin.

Subsequent increases in recruitment of tight junction proteins and signalling proteins such guanine nucleotide exchange factors that activate signalling by RhoA and Cdc42 induces junctional maturation, which involves the formation of distinct tight and adherens junctions, and a junctional enrichment of the actomyosin cytoskeleton. Myosin activation promotes the development of regular epithelial cell shapes (e.g., columnar epithelia such as those in the intestinal tract. Finally, polarisation is then induced by polar activation of Cdc42 along the apical domain and at the marginal zone close to tight junctions. Active Cdc42 binds to the PAR3-PAR6-aPKC complex, leading to activation of the kinase, and induces development of a polarized cell surface with a welldifferentiated apical cell membrane (e.g., a brush border membrane in intestinal and many other epithelial cells). (B) Complexes involved in cell adhesion are signal hubs that send and receive signals that guide cell behaviour, function and morphogenesis; extensive crosstalk exists between different adhesion complexes. For example, JAMs are recruited by forming adherens junction via interactions mediated by the tight junction protein $\mathrm{ZO}-1$ and the nectin binding protein AF- 6 . This then leads to the increased recruitment of other junctional proteins and activation of two small GTPases, Rap1 and Rap2, that regulate the functions of integrin-based focal adhesions and of adherens junctions. Forming tight junctions also recruit activators of Rho GTPases, guanine nucleotide exchange factors like GEF-H1, which is inactive at junctions, and p114RhoGEF ( $p 114 R G$ ), which drives junctional RhoA activation, due to interactions with junctional adaptor complexes formed by cingulin and JACOP, which are recruited by ZO-1. Tight junction dissociation triggers the release of GEF-H1 leading to RhoA activation along the base of the cells. This stimulates the induction of stress fibres and increased focal adhesion formation including the recruitment of proteins that regulate focal adhesions such as focal adhesion kinase (FAK). In endothelial cells, ZO-1 coordinates junctional actomyosin activity leading to increased cell-cell tension and pulling on cadherin-based adherens junctions via a molecular bridge between the actomyosin cytoskeleton and the cadherin formed by $\alpha$ - and $\beta$-catenin, as well as vinculin. Vinculin can be recruited to both adherens junction, by interaction with $\alpha$-catenin, and focal adhesions, by 
interaction with talin and $\alpha$-actinin, which is regulated by cytoskeletal tension pulling on an adhesion complex. Claudin-7 and -11 have been reported to form complexes with integrins and regulate migration; however, it is not yet clear whether this indeed represents crosstalk between tight junctions and focal adhesions, or occurs independently of tight junctions. 
Signalling to tight junctions: Regulation of junction assembly and function

- JAMA - PAR3/PAR6/aPKC; initiation of junction assembly

- Crb3 - Pals1, PATJ, ZO-3 - aPKC; junction assembly and initiation of polarisation

- Tricellulin, ZO-1 - TUBA; stimulation of Cdc42/N-WASP regulated F-actin organisation

- JAMA, ZO-1, JACOP (Paracingulin) - p114RhoGEF; regulation of RhoA/ROCKII/myosinll and endothelial cell-cell tension

- ZO-1 - ARHGEF11; stimulation of RhoA and myosinll activation

- Cingulin, PATJ - p114RhoGEF; stimulation of RhoA/ROCKII/myosinll, regulated by Lulu2

- PAR3, PAR6 - ECT2; activation of Cdc42/aPKC signalling

- JACOP (Paracingulin) - SH3BP1; negative regulation of Cdc42, regulation of actin remodelling

- Angiomotin - $\mathrm{RICH} 1$; negative regulation of Cdc42

- ZO-1 - heterotrimeric G proteins (e.g., Gai2); regulation of junction assembly

- aPKC, PP2A; regulation of TJ protein phosphorylation

- WNK1/4 - Claudins; regulation of paracellular ion conductance

- AMP-activated Kinase (stimulated by LKB1 and Calmodulin activated Kinase II); stimulation of junction assembly, phosphorylation of claudins and cingulin

- classical and novel PKCs and PKA; stimulation of junctional cytoskeleton, assembly and function

Signalling from tight junctions: Cell behaviour, survival and differentiation

- Crb3, Pals1, PAR6, PAR3 - aPKC; apical differentiation

- MARVELD3 - MEKK1; regulation of JNK signalling, gene expression and stress response

- Occludin - c-yes, Raf-1 kinase, PI3 Kinase, TGF $\beta R 1$, c-src, E3 ubiquitin-protein ligase Itch; regulation of cell transformation and junction dissociation

- JAM-A, tetraspanin (CD9)/integrin complexes; angiogenic signalling and migration

- Ezrin - Dbl3 and Cdc42; apical differentiation

- Cingulin, Jacob (paracingulin) - GEF-H1; downregulation of cytoplasmic RhoA signalling and stress fibres

- ZO-1 - ZONAB/symplekin/CDK4, GEF-H1, Apg-2; transcriptional and posttranscriptional regulation of gene expression, proliferation, stress response and survival

- ZO-2 - c-Myc, SAFB, AP-1; regulation of gene expression and proliferation

- Merlin, Angiomotin, PAR3, Mst, LATS, ZO-1/2 - YAP and TAZ; regulation of gene expression and proliferation

- Angiomotin - $\mathrm{RICH} 1$; regulation of Rac-activated MAP kinase signalling

- PATJ - Tsc2; regulation of mTORC1 activity

- MAGI1/2/3 - PTEN; regulation of Akt and cell survival signalling

Tight junctions are connected to the main cellular signalling networks that guide cell shape and junction assembly, transcriptional and posttranscriptional gene expression, and cell proliferation and differentiation. These signalling mechanisms transmit information in two directions: Form the cell interior to the junction to guide junction assembly and function, and from the junction to the cell to guide gene expression, proliferation, and differentiation. Summarised are the main signalling mechanisms linked to TJ and their main components. Adaptor proteins are blue, transmembrane proteins brown, and signalling proteins red. The hyphen refers to regulatory links that are generally mediated by direct protein/protein interactions. 


\section{Box 1 - Evolutionary conservation of tight junction functions}

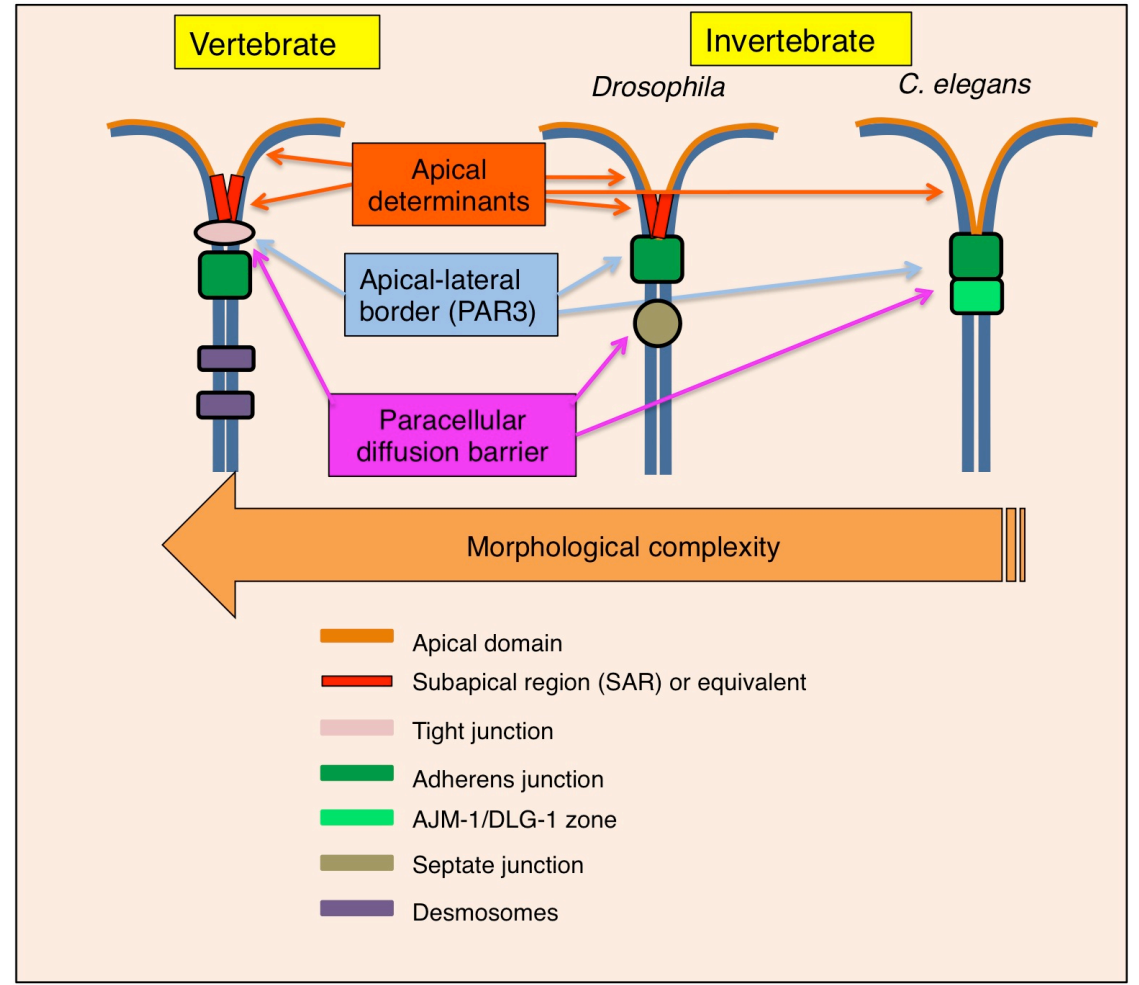

Occluding junctions show a higher degree of variation in metazoans than cadherin-based junctions. In vertebrates, tight junctions are located apical to adherens junctions, whereas in invertebrates the most apical junctional structures are commonly adherens junctions. Although tight junction structures exist in some lower invertebrates and chordates, the equivalent structure in many invertebrate epithelia is located basal to the adherens junction, and is known as septate junction (e.g., insects), or the diffusion barrier may be part of adherens junctions (e.g., C. elegans) 171,172. The septate junction in Drosophila contains claudin-like molecules that are important for barrier function ${ }^{173-177}$. C. elegans also expresses claudin-like molecules, and at least two of them are important for barrier formation; however, they are associated with adherens junctions ${ }^{178}$. Thus, the importance of claudins for barrier formation is conserved, the junction they associate with is not.

Another striking example of evolutionary conservation is the machinery associated with apical polarization. Tight junctions form the apical/lateral border in vertebrates whereas this border is associated with adherens junctions in invertebrates. Just apical to the apical/lateral border is a specialized signalling zone, the subapical region (SAR; also known as apical marginal zone) that was first identified in Drosophila. The signalling mechanisms that regulate apical polarization include two protein complexes formed by apical determinants: the PAR-3/PAR-6/aPKC/Cdc-42 and the Crb3/Pals1/PATJ complexes. Both complexes have been reported to associate with tight junctions in vertebrates and have homologs in C. elegans and Drosophila, where they associate with the SAR ${ }^{179}$. The evolutionary conservation may even extend further as a SAR-like signalling zone enriched in aPKC, Crb3, ezrin and the Cdc42 activator Dbl3 is also associated with the apical end of tight junctions in vertebrates ${ }^{125}$.

Different junctional functions are thus associated with different types of junctions in different phyla, suggesting that intercellular junctions have become reconfigured during evolution but that individual processes are conserved. This is also reflected in the molecular remodelling that occurs 
during junction assembly: an initial primordial adhesive complex contains components of tight junctions and adherens junctions, and then matures into distinct junctional complexes ${ }^{180}$. 


\section{Box 2 - Tight junctions and molecular links to human disease}

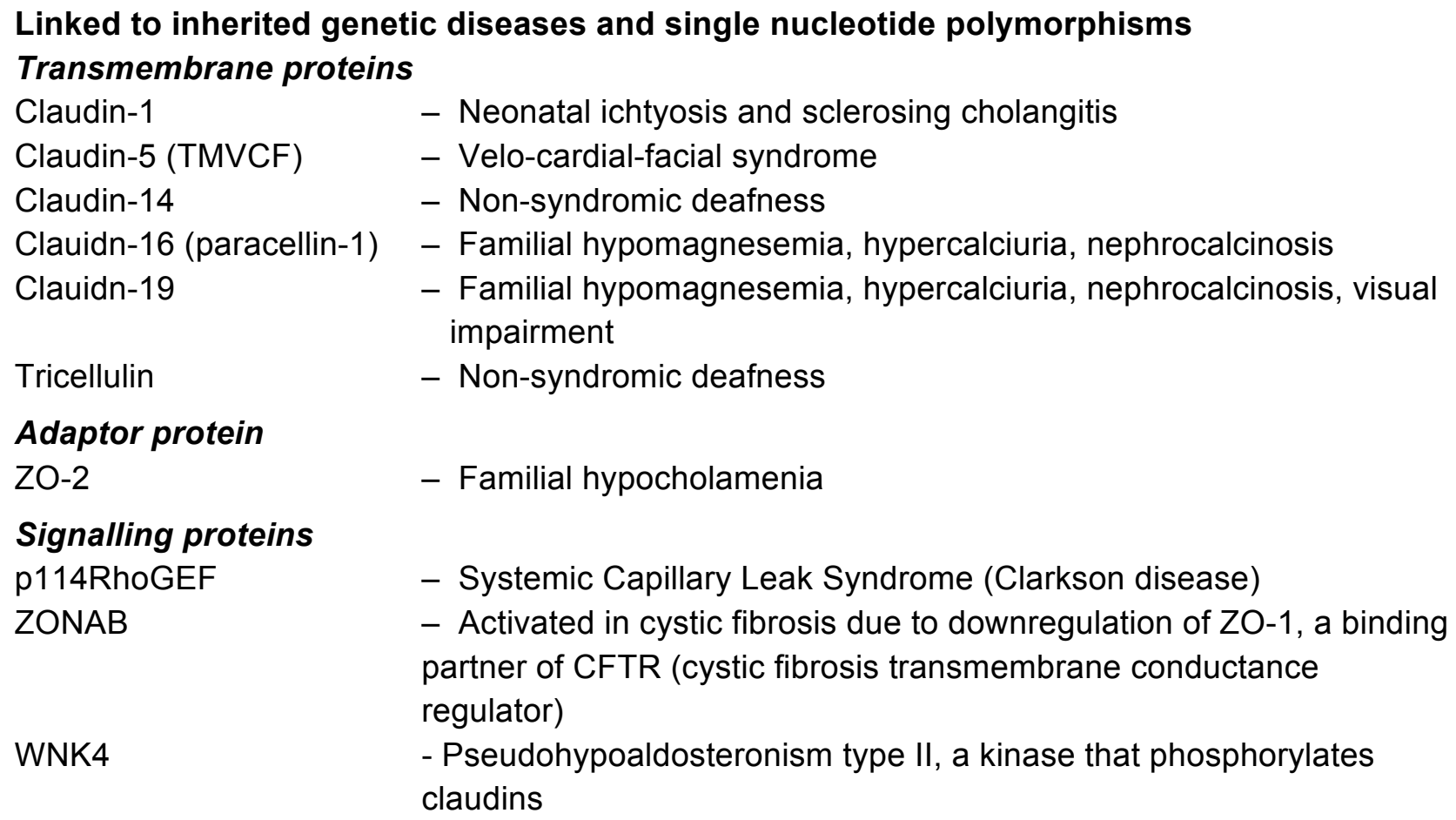

Tight junction components targeted by pathogenic viruses and bacteria

Transmembrane proteins

Claudin-1/6/9; occludin - Hepatitis C virus, infection

$\begin{array}{ll}\text { JAM-A } & - \text { Reovirus, infection } \\ \text { CAR } & - \text { Coxsackie virus, infection } \\ \text { Claudin-3/4 } & - \text { Clostridium, junction dissociation } \\ \text { Occludin } & - \text { V. cholera, junction dissociation }\end{array}$

Adaptor proteins

ZO-1, ZO-2 - Tick-borne encephalitis and Dengue viruses

ZO-2, MUPP1, PATJ, - Adenovirus

MAGI1

MAGI1-3, PATJ, - Papillomaviruses, papilloma formation

MUPP1, PAR3

MAGI1-3

- Influenza A virus, junction dissociation

Pals1

- Severe acute respiratory syndrome virus, retarded junction formation

\section{Signalling proteins}

GEF-H1

- H. pylori via PAR1, junction dissociation, leads to displacement of structural junctional proteins like ZO-1 and occludin

TJs have been linked to diseases that affect many tissues and organs. Some of these diseases are inherited and involve mutations or polymorphisms in TJ-associated proteins themselves or lead to activation of TJ-associated signalling mechanisms as in the case of cystic fibrosis. Similarly, multiple pathogenic viruses and bacteria are known to target TJ. Usually, this involves direct interactions with junctional proteins but, as in the case of $\mathrm{H}$. pylori, may involve activation of a cellular signalling protein that is not associated with TJ, PAR1, that then stimulates a junctional signalling pathway. Many other diseases such as chronic inflammatory conditions and cancer have 
been linked to $\mathrm{TJ}$ but it is generally not known whether $\mathrm{TJ}$ deregulation is a cause or consequence of disease. For more details, see ${ }^{43,85}$. 